\title{
PRIMITIVE GROUPS HAVING TRANSITIVE SUBGROUPS OF SMALLER, PRIME POWER DEGREE ${ }^{\dagger}$
}

BY

\author{
WILLIAM M. KANTOR
}

ABSTRACT

The groups in the title are classified, provided they are not too highly transitive.

Let $G$ be a primitive permutation group on a finite set $S$ of $n$ points. In 1871, Jordan initiated the study of $G$ under the additional assumption that there is a transitive subgroup $H$ of degree $m$, where $1<m<n$; that is, $H$ fixes $n-m$ points and is transitive on the remaining points.

THEOREM 1. If $m$ is a prime power, and $G$ is not $n-m+1$-transitive, then $G$ is one of the following groups in its usual 2-transitive representation: a collineation group of $\operatorname{PG}(d-1, q)$ containing $P S L(d, q)$, where $d \geqq 3$; the full collineation group of $A G(d, 2)$, where $d \geqq 3$; or a Mathieu group $M_{22}$, $\operatorname{Aut}\left(M_{22}\right), M_{23}$, or $M_{24}$.

This result contains several recent theorems found in [2], [3] and [4].

Proof. $G$ is 2-transitive on $S$ ([7, p. 32]). By [2, Sect. 6], we may assume that $G$ is not 3-transitive. Let $B$ be the complement of the given set of $m$ points, so $|B|=k=n-m$. Let $P$ be a Sylow subgroup of the pointwise stabilizer $G(B)$ of $B$, so that $P$ is transitive on $S-B$. By [2, (3.6)], the distinct sets $B^{g}$, $g \in G$, form a design $\mathscr{D}$ whose lines have more than two points; moreover, if $B$ is not a line of $\mathscr{D}$, then planes of $\mathscr{D}$ are well-defined and $G$ is transitive on the set of planes.

Suppose first that $B$ is a line of $\mathscr{D}$. Let $g \in G$ be such that $B \cap B^{g}$ is a point $x$. Since $P$ is transitive on the lines $\neq B$ on $x$, the stabilizer $P_{1}$ of $B^{g}$ in $P$ has index

$\dagger^{\top}$ This research was supported in part by NSF Grant GP 37982 X.

Received January 1, 1974 
$(n-k) /(k-1)<|S-B|$ in $P$. Hence, $P_{1}$ fixes no point of $S-B$. We may assume that $P_{1}$ normalizes $P^{g}$, and hence centralizes some $z \neq 1$ in $Z\left(P^{g}\right)$. Then $B^{z}=B$ as $B$ is the set of fixed points of $P_{1}$. However, $P^{g}$ is transitive on the lines $\neq B^{g}$ on $x$, so $z$ must fix each line on $x$. Also, $z$ fixes each point of $B^{g}$. Consequently, by a theorem of O'Nan [5], $\mathscr{D}$ consists of the points and lines of a projective space $P G(d-1, q), d \geqq 3$, and $G$ contains $P S L(d, q)$. Since $|S-B|$ is a prime power, $d=3$.

Now suppose $B$ is not a line of $\mathscr{D}$, and let $E$ be any plane meeting $B$ in a line. Then the sets $(E-B \cap E)^{h}, h \in P$, partition $S-B$, so $|E-B \cap E|$ is a prime power. By [2, (3.10)], the global stabilizer of $E$ in $G$ induces on $E$ a group inheriting our hypotheses. Thus, each plane is a projective plane, so the points and lines of $\mathscr{D}$ form a projective space $P G(d-1, q)$ (see Veblen and Young [6]). Also, $B$ is a subspace, and hence a hyperplane since $|S-B|$ is a prime power. Consequently, $G \geqq P S L(d, q)$.

By using a slightly more complicated argument (depending more heavily on [2]), we can generalize Theorem 1 as follows.

THEOREM 2. Suppose $G$ is a finite group primitive on a set $S$ of $n$ points. Let $B \subset S$, where $|B|=k<n-1$, and assume that $G$ is not $k$-transitive. Then $G$ is as in Theorem 1 if either of the following holds for the pointwise stabilizer $G(B)$ of $B$ :

(i) $G(B)$ has a nilpotent Hall subgroup transitive on $S-B$; or

(ii) There is a prime $p \mid k-\mu$, where $\mu=\max \left\{\left|B \cap B^{g}\right| \mid B \neq B^{g}, g \in G\right\}$, and a Sylow p-subgroup $P$ of $G(B)$, such that $C_{G(B)}\left(\Omega_{1}(Z(P))\right.$ is transitive on $S-B$.

Here, as usual, $\Omega_{1}(Z(P))=\left\{g \in Z(P) \mid g^{p}=1\right\}$.

We will only sketch the proof, which is similar to that of Theorem 1 . We will assume familiarity with [2, Sect. 3]. Since $1<k-\mu \mid n-k$ for Jordan groups, (i) is actually a special case of (ii). Thus, assume (ii). We may assume that $G$ is not 3-transitive. Let $\mathscr{L}$ denote the (geometric) lattice of intersections of families of blocks.

Fix $F=B \cap C \in \mathscr{L}$ with $B$ and $C$ blocks and $|F|=\mu$. Let $P$ be a Sylow $p$-subgroup of $G(B)$, and $P_{1}$ the stabilizer in $P$ of $C$. Since $p \mid k-\mu, P_{1}$ fixes no point of $S-B$. Also, $P_{1}$ normalizes a Sylow $p$-subgroup $Q$ of $G(C)$, and hence centralizes some $z \neq 1$ in $\Omega_{1}(Z(Q))$. Since $C_{G(C)}(z)$ is transitive on $S-C$, while $z$ fixes $B$, it follows that $z$ fixes all blocks containing $F$. 
Choose $Y \in \mathscr{L}$ such that $Y \subseteq F, z$ fixes all blocks containing $F$, and $Y$ is minimal with respect to these conditions. Then $Y \neq \varnothing$, so we can choose $X \in \mathscr{L}$ maximal in $Y$ (where possibly $X=\varnothing$ ). Note that $G(X)$ is 2-transitive on $S(X)$ $=\left\{Y^{g} \mid X \subset Y^{g}, g \in G\right\}$. By [5], $S(X)$ is the set of points of a projective space, on which $G(X)$ induces at least the projective special linear group. Now the theorem follows from [2, Sect. 6].

Finally, we remark that it is very easy to use [5] to prove Theorem 2 if (i) and (ii) are replaced by the condition that $G(B)$ has an abelian subgroup transitive on $S-B$.

\section{REFERENCES}

1. P. Dembowski, Finite Geometries, Springer, Berlin-Heidelberg-New York, 1968.

2. W. M. Kantor, Jordan groups, J. Algebra 12 (1969), 471-493.

3. T. P. McDonough, On Jordan groups, J. London Math. Soc. 6 (1972), 73-80.

4. T. P. McDonough, On Jordan groups - addendum, to appear.

5. M. E. O'Nan, The normal structure of the one-point stabilizer of a doubly-transitive permutation group, $I$, to appear.

6. O. Veblen and J. W. Young, Projective Geometry I, Ginn, Boston, 1910.

7. H. Wielandt, Finite Permutation Groups, Academic Press, New York, 1964.

Department of Mathematics

UNIVERSITY OF OREGON

EuGENE, OREGon, U. S. A. 